D) Check for updates

Cite this: RSC Adv., 2017, 7, 29882

DOI: $10.1039 / c 7 r a 90068 f$

www.rsc.org/advances

\section{Correction: Punica granatum (pomegranate) peel extract exerts potent antitumor and anti-metastasis activity in thyroid cancer}

\author{
Yujue Li, ${ }^{a}$ Tinghong Ye, ${ }^{b}$ Fangfang Yang, ${ }^{\mathrm{b}}$ Mingxing $\mathrm{Hu},{ }^{\mathrm{b}}$ Libo Liang, ${ }^{\mathrm{a}} \mathrm{He} \mathrm{He},{ }^{\mathrm{c}}$ \\ Zhipeng Li, ${ }^{a}$ Anqi Zeng, ${ }^{\text {b Yali Li, }}$, Yuqin Yao, ${ }^{\text {Y Yongmei Xie, }}$ ' Zhenmei An*a \\ and Shuangqing $\mathrm{Li}^{\star a}$
}

Correction for 'Punica granatum (pomegranate) peel extract exerts potent antitumor and anti-metastasis activity in thyroid cancer' by Yujue Li et al., RSC Adv., 2016, 6, 84523-84535.

The authors regret that the values provided for the content of punicalagin (PC) and ellagic acid (EA) in pomegranate peel extract (PoPx) were incorrect in the original article. The correct values are $83.9 \mathrm{mg} \mathrm{g}^{-1}$ for PC and $7.3 \mathrm{mg} \mathrm{g}^{-1}$ for EA. Two sentences should be revised described below.

In the Results section, subsection 'The content and molecular weight of punicalagin and ellagic acid in PoPx', the final sentence should be revised as follows:

"The results of the analysis reveal that PC accounts for $83.9 \mathrm{mg} \mathrm{g}^{-1}$ and EA accounts for $7.3 \mathrm{mg} \mathrm{g}^{-1}$ in PoPx." In the Discussion section on p. 84533, the second sentence of the last paragraph should be revised as follows: "We firstly detected the content of PC and EA in PoPx, which was $83.9 \mathrm{mg} \mathrm{g}^{-1}$ and $7.3 \mathrm{mg} \mathrm{g}^{-1}$, respectively." The Royal Society of Chemistry apologises for these errors and any consequent inconvenience to authors and readers.

${ }^{a}$ Department of Endocrinology and Metabolism, Department of General Practice Medicine, West China Hospital, Sichuan University, Chengdu, China. E-mail: lsqhxjk@126.com; anzm1997@sina.com; Fax: +86 28 85164060; Tel: +86 2885503817

${ }^{b}$ State Key Laboratory of Biotherapy and Department of Liver Surgery, West China Hospital, Sichuan University and Collaborative Innovation Center for Biotherapy, Chengdu, China

'Department of Laboratory Medicine, West China Hospital, Sichuan University, Chengdu, China 